\title{
Measuring weight and location of individual bites using a sensor augmented smart plate
}

\author{
Gert Mertes $^{1,2}$, Glenn Christiaensen ${ }^{1}$, Hans Hallez $^{3}$, Sammy Verslype ${ }^{3}$, Wei Chen $^{4}$ and Bart Vanrumste ${ }^{1,2}$
}

\begin{abstract}
In this work, a novel plate system that can detect weight and location of individual bites during meals is presented. The system consists of a base station with sensors and a detachable off-the-shelf polymer plate with three compartments. By combining data from multiple weight sensors, the weight of individual bites can be accurately measured and localized on the plate to determine the compartment from which they were taken. With prior knowledge of the weight of the food in each compartment at the start of the meal, the system can estimate the nutritional value of the consumed food. In a test conducted in a controlled home environment, the system was able to measure the weight of consumed food in each compartment with a maximum relative error of $1.4 \%$. The goal of the system is to replace traditional monitoring tools and to automatically monitor the amount of consumption.
\end{abstract}

\section{INTRODUCTION}

Accurately measuring food intake is a requirement in several health care environments, such as the treatment of eating disorders, obesity and monitoring of food intake for elderly people in both a hospital and home environment. Traditional methods typically consist of pen-and-paper tools such as questionnaires and food diaries [1]. These are often incomplete due to their time consuming nature and incorrect due to human error or deliberate misreporting of the food intake. A potential solution to this problem is the use of a monitoring system that automatically monitors a user's food intake. Eating activity can be detected using various wearable devices. Using a wearable microphone, the sounds related to eating activity, such as chewing, can be automatically distinguished from other activities [2], [3]. Using wearable accelerometers, eating activity can be detected from characteristic movements of the body during eating activity [4]-[6].

Measuring the amount of food can be done by integrating weight sensors into the eating surface of the subject. This method was first proposed by Kissileff et al. in 1980 [7]. Several modern adaptations to this approach have been proposed since then [8], [9]. These devices can detect the overall consumed food and measure individual bites, but lack the ability to estimate the type of food consumed. Furthermore, existing methods require either extensive adaptation of the existing eating surface or are big and not easily adoptable.

In this work, we propose a novel smart plate system with a low hardware footprint. It can accurately detect individual bites and estimate the position from where the bite was taken.

This work is funded by DISH and Engineers@CareHome, supported by KU Leuven, Vlaams Brabant and OCMW Leuven.

${ }^{1} \mathrm{KU}$ Leuven, Campus Groep T, e-Media Lab, Belgium

${ }^{2} \mathrm{KU}$ Leuven, ESAT-STADIUS-IMEC, Belgium

${ }^{3} \mathrm{KU}$ Leuven, Campus Brugge, M-Group, Belgium

${ }^{4}$ Fudan University, Center for Intelligent Medical Electronics, China

\section{HARDWARE}

\section{A. Mechanical Design}

A custom acquisition system that integrates with an existing off-the-shelf eating plate was developed, shown in Figure 1. The system consists of a base station that contains all the hardware. The polymer eating plate is mounted and rests on top of weight sensors in the base station. Load cells are used to accurately measure changes in weight. A load cell is a mechanical structure that can measure weight by measuring the deformation of the structure when a mass is placed onto it. These sensors are commonly used in accurate weight scales. The base station is circular in shape to fit most common eating-plates. Currently, however, the base station only accepts one type of plate, but this can be expanded in the future. Three load cells are mounted on the base station, placed at $120^{\circ}$ intervals around the circumference of the base, as shown in Figure 2. One side of the load cell is permanently fixed to the base station while the other end is free floating. The free floating part of the cell mates up with mounting points at the bottom of the polymer plate. The polymer plate is not permanently fixed to the load cells. It is kept in place using a reversible friction-fit connection. This allows for easy removal of the plate for cleaning, while ensuring that the plate stays in the same position and orientation when in use. The load cells are of the type CZL616C. This type of cell has a small footprint with a height of $6 \mathrm{~mm}$, which allows for a low-profile base station. The cells have a rated capacity of $780 \mathrm{~g}$. Since the weight of the plate is spread out over three load cells, this allows for a rated capacity of $2.3 \mathrm{~kg}$. The polymer plate has a mass of $400 \mathrm{~g}$, which allows for a maximum meal weight of $1.9 \mathrm{~kg}$ while keeping a buffer available for physical interaction with the plate, such as pushing into the plate while cutting. This should be sufficient for most, if not all, meals. While this is highly dependent on the type of food being consumed, meal weight never exceeded $600 \mathrm{~g}$ during our testing, even with the plate completely full.

\section{B. Electrical Design}

A custom embedded system was designed around an Atmel ATmega644 MCU to capture and store data from the load cells. The system is powered by a Li-ion battery and can be charged via USB. Sensor data is stored locally on a SD card. A real-time clock module allows synchronization with an external clock. There is a Bluetooth module for wireless communication with the system. The wireless link can be used to configure the device and to execute commands 


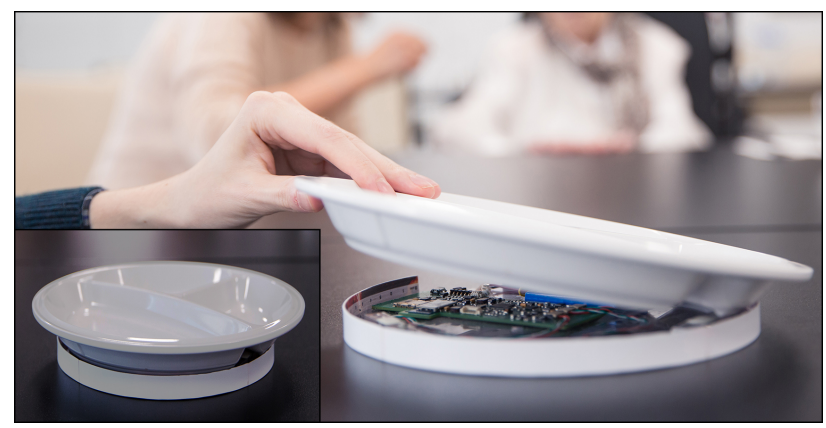

Fig. 1: The smart plate system: base station and plate.

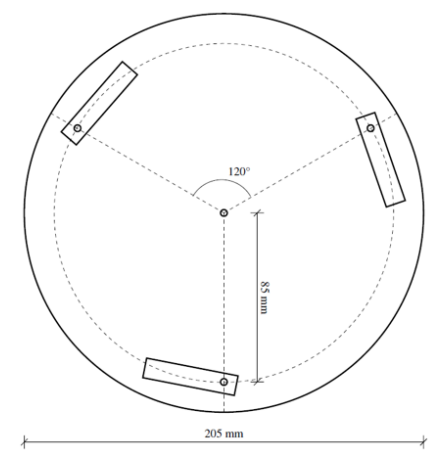

Fig. 2: Orientation of the load cells in the base station.

such as starting or stopping a measurement. There are four channels available for respectively four load cells. Each channel is amplified by an AD623 instrumentation amplifier. Sampling is done by an ADS1224 24-bit, 4-channel ADC. Only three channels are currently occupied to accommodate a round plate. The sampling rate can be selected at $6 \mathrm{~Hz}$, $12 \mathrm{~Hz}$ or $24 \mathrm{~Hz}$. The resolution and accuracy of the system is $0.1 \mathrm{~g}$ and $0.3 \mathrm{~g}$ respectively.

\section{METHODOLOGY}

\section{A. Bite Localization}

The location of each bite can be derived from the plates' center of mass. Assuming three load cells, the coordinates $\boldsymbol{R}$ of the center of mass can be calculated relative to a chosen origin using formula 1. It is derived from the force and momentum balance equation of the plate resting on the load cells, with $F_{\text {tot }}$ being the amplitude of the total force acting on all load cells, $F_{i}$ the force on sensor $i$ and $\boldsymbol{r}_{\boldsymbol{i}}$ the distance vector from the origin to sensor $i$.

$$
\boldsymbol{R}=\frac{1}{F_{t o t}} \sum_{i=1}^{3} F_{i} \boldsymbol{r}_{\boldsymbol{i}}
$$

When a bite is taken from the plate at a certain position, $\boldsymbol{R}$ will shift in the opposite direction with a distance proportional to the bite weight. By calculating $\boldsymbol{R}$ before and after the bite, the bite can be localized. Formula 1 can be rewritten for this purpose. This results in equations 2 and 3 , with $x$ and $y$ the bites' location, $\Delta F_{i}$ the change in force on sensor $i$ from before to after the bite, $\Delta F_{\text {tot }}$ the total change in force on all sensors and $x_{i}$ and $y_{i}$ the distance from the origin to sensor $i$.

$$
\begin{aligned}
& x=\frac{1}{\Delta F_{\text {tot }}} \sum_{i=1}^{3} \Delta F_{i} x_{i} \\
& y=\frac{1}{\Delta F_{\text {tot }}} \sum_{i=1}^{3} \Delta F_{i} y_{i}
\end{aligned}
$$

The above equations were tested by placing a mass at known positions on the plate. A mass of $5 \mathrm{~g}$ was chosen to reflect a realistic bite size. The result is shown in Figure 3 . The blue dots are the sensor positions, the red crosses indicate the detected locations, while the crossings of the grid lines indicate the actual position. Also shown is the measured weight and the error in $\mathrm{mm}$ for each detection, calculated as the Euclidean distance between the detected and actual position. The average error is $5.16 \mathrm{~mm}$. This error is due to the limited accuracy of the load cells. While the individual cells have a precision of $0.1 \mathrm{~g}$, this measurement error accumulates in formulas 2 and 3. For small masses, this can result in a location error of several millimeters, but since the walls of the compartments in the plate are at least $10 \mathrm{~mm}$ thick, the error is small enough to accurately detect the compartment of each bite.

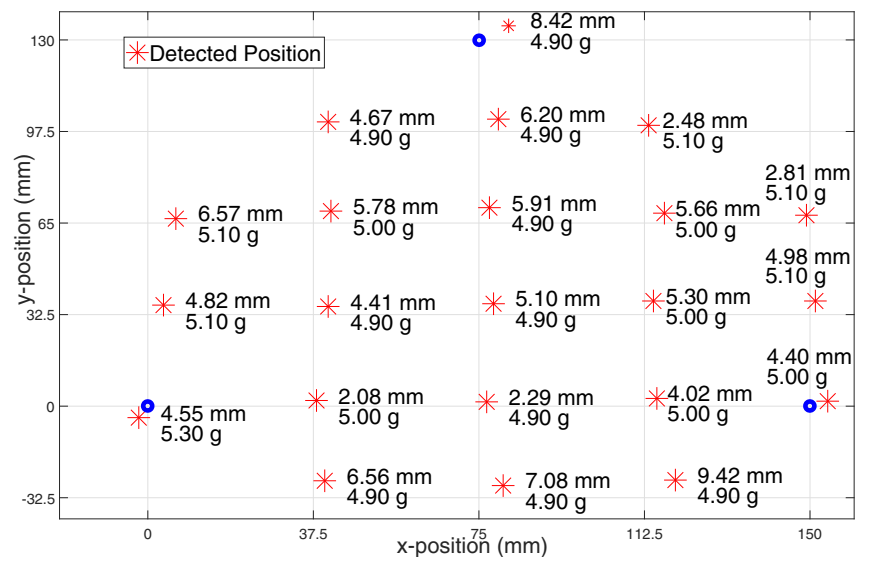

Fig. 3: Accuracy of the position detection. The blue dots indicate the sensor locations.

\section{B. Step Detection}

As discussed in the previous section, the force on the load cells before and after the bite should be known in order to detect the bites' location. A bite typically results in a sudden decrease in weight in the raw sensor data. As such, a step detection algorithm can be used to detect the time of each bite, after which the force before and after the bite can be found and the location calculated. To begin with, the raw signals from the three load cells are added together. After calibration and subtracting the weight of the plate, this signal immediately gives us the total weight of the food. The signal is then filtered with a moving median filter to discard noise and outliers. The goal is to filter out any movement prior to each bite that may be caused by pressing down on the 


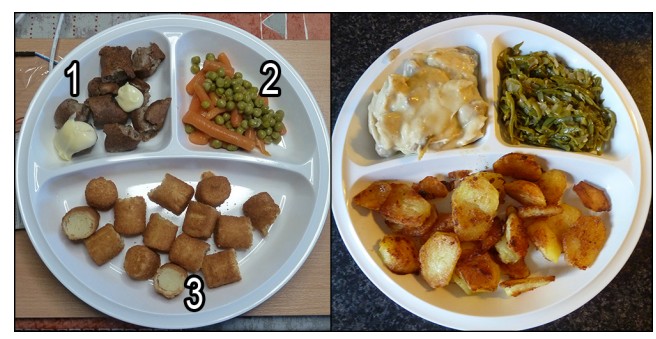

Fig. 4: The test setup of the lab (left) and home (right) measurements. The compartments are numbered 1 to 3 .

plate or movements such as cutting or scooping. Steps are subsequently detected by calculating the local maxima of the first derivative of the filtered signal. The local maxima are thresholded so that only sufficiently big steps are regarded as a bite. The size of a step is subsequently calculated by subtracting the mean value of all samples after the step (up until the next step) from the mean value of all samples before the step (up until the previous step).

\section{Data Collection}

To show the feasibility of the proposed approach, data was collected from two test subjects. Meals were consumed using the smart plate system and sampled at $6 \mathrm{~Hz}$. One subject was asked to consume a meal in a lab environment and the other subject was asked to consume a meal in a home environment. Figure 4 shows the start situation for each measurement. Food was pre-cut in both cases and subjects were only allowed to use a fork. For the lab measurement, the subject was asked to eat slowly and take care to minimize actions that would introduce large amounts of movement or pressure, such as overly pushing into the plate. For the home measurement, the subject could eat at their own speed and no restrictions were placed on how the food was picked up. Subjects and the plate were video recorded synchronously to the smart plate to validate the detected bites. This was done by synchronizing the plate's real time clock with the video capture system clock at the start of each measurement. The drift of the plate's clock is less than $0.5 \mathrm{~s}$ for the duration of the measurement, which is sufficient to verify detected bites.

\section{RESULTS}

\section{A. Bite Location Detection}

Table I shows the results of the bite localization for both measurements. Figure 5 shows a graphical representation of all detected bites in the lab measurement. The compartment of each bite can be determined by overlaying the detection results on top of the plate layout. Figure 4 shows the compartment numbers. Almost all of the bites are detected. In the lab measurement, one bite that was supposed to be detected in compartment 1 , is detected in compartment 2 . This kind of error can occur when a bite is taken near the wall that divides both compartments. A similar situation occurs in the results of the home measurement. A bite that was taken from compartment 3 is detected in compartment 1. Additionally, two bites remain undetected from compartment 3 . This can
TABLE I: Bite localization results per compartment.

Lab data

\begin{tabular}{l|c|c|c} 
& Real Bites & Detected Bites & \# Errors \\
\hline Compartment 1 & 9 & 10 & 1 \\
\hline Compartment 2 & 11 & 10 & 1 \\
\hline Compartment 3 & 14 & 14 & 0
\end{tabular}

Home data

\begin{tabular}{l|c|c|c} 
& Real Bites & Detected Bites & \# Errors \\
\hline Compartment 1 & 8 & 9 & 1 \\
\hline Compartment 2 & 8 & 8 & 0 \\
\hline Compartment 3 & 11 & 9 & 2
\end{tabular}

happen when the subject takes a bite and immediately takes another bite without allowing the weight-signal to stabilize (e.g.: taking two bites while still manipulating the plate with the free hand). This can result in multiple bites being aggregated into one. When bites from the same compartment are aggregated together, however, the total weight of the compartment will still be correct, which is the case in the home measurement.

\section{B. Bite Weight Detection}

Each bite now has a weight and a compartment assigned to it. The total weight of food taken from each compartment can thus be determined by summing the weights of all bites detected in each compartment. The results are shown in Table II for both measurements. The real weight of the food in each compartment was measured with a kitchen scale before the start of the meal. The total weight is the sum of the weights of each compartment. For the lab and home measurement, the total detected weight only differs $0.1 \%$ and $0.6 \%$ respectively from the real weight.

Looking at the detected weights of individual compartments, the home measurement performs better than the lab measurement. This, however, is a coincidence. In the lab measurement, only a single bite was detected in the wrong location, causing an error in both the detected and the real compartment. Since compartment weights are relatively small, a single wrong-detected bite can have a substantial impact on the relative error. The total weight, however, remains correct as the size of all bites was detected correctly. For the home measurement, multiple subsequent bites from compartment 3 were aggregated together in a single bite, but because all bites were taken from the same compartment, the total weight for compartment 3 remains correct.

\section{DISCUSSION AND OUTLOOK}

The test results show that the system is capable of detecting the total amount of food taken from each compartment with a precision in the grand order of several grams. This is sufficient to estimate the total amount of calories, given that the food present on the plate is known beforehand. In situations where food monitoring may be required, such as treatment of eating disorders, hospitalization or elderly care, food is typically served from a central kitchen with a known menu. The caloric value of the full meal is often already 
TABLE II: Bite weight detection results per compartment.

Lab data

\begin{tabular}{c|c|c|c} 
& Real Weight & Detected Weight & Rel. Error \\
\hline Compartment 1 & $77.1 \mathrm{~g}$ & $80.5 \mathrm{~g}$ & $4.4 \%$ \\
\hline Compartment 2 & $62.9 \mathrm{~g}$ & $56.8 \mathrm{~g}$ & $9.7 \%$ \\
\hline Compartment 3 & $137.2 \mathrm{~g}$ & $139.8 \mathrm{~g}$ & $1.9 \%$ \\
\hline \hline Total Weight & $277.2 \mathrm{~g}$ & $277.1 \mathrm{~g}$ & $0.1 \%$
\end{tabular}

Home data

\begin{tabular}{c|c|c|c} 
& Real Weight & Detected Weight & Rel. Error \\
\hline Compartment 1 & $115.0 \mathrm{~g}$ & $114.6 \mathrm{~g}$ & $0.3 \%$ \\
\hline Compartment 2 & $102.4 \mathrm{~g}$ & $100.9 \mathrm{~g}$ & $1.4 \%$ \\
\hline Compartment 3 & $64.9 \mathrm{~g}$ & $65.0 \mathrm{~g}$ & $0.2 \%$ \\
\hline \hline Total Weight & $282.3 \mathrm{~g}$ & $280.5 \mathrm{~g}$ & $0.6 \%$
\end{tabular}

known beforehand. This information can be combined with the output of our system to accurately measure the intake.

Our test results, however, were obtained with data captured in a controlled environment. The lab measurement was heavily constrained with a minimum amount of interaction with the plate. The home environment was less constrained, but the meal was consumed in a calm manner, which is highly dependent on the test subject, resulting in relatively clean data in which bites can easily be detected. While the home measurement also had pre-cut foods and the subject could only use a fork, it remains a realistic scenario. In situations where food monitoring is prevalent, especially in elderly care, pre-cut and ready-to-eat food is common.

The step detection algorithm, however, is not yet suited for use in uncontrolled environments. The biggest challenge during unrestricted eating is distinguishing bites from other activities such as cutting, re-arranging food or resting utensils on the plate. Care should also be taken to detect situations where users pick up a piece of food and then return it to the plate, potentially half-eaten. Mattfeld et al. propose an algorithm that detects epochs of stability combined with constraints to solve this problem [9]. If a change in weight is observed between subsequent stable epochs, this is regarded as a bite. The idea behind this approach is similar to the moving median filter used in our algorithm. Furthermore, a set of constraints is defined by which bites should comply. For example, after a detected bite, the weight may not increase. If this is the case, this can indicate a half eaten-bite or the user resting utensils on the plate. While the algorithm proposed by Mattfeld et al. is built with unrestricted eating in mind, only $39 \%$ of bites were correctly detected. Further improvement of the algorithm could be a part of future work.

Our system has the advantage that it can also detect location and estimate the amount of calories without external sensors. Furthermore, it can also be used to extract parameters such as bite size or eating speed. Typical methods of measuring caloric intake require the user to keep a detailed food diary. Camera based systems can be used to estimate the amount of food using image based methods, but require usage of a smartphone or a camera system in the eating area [10]-[12]. Zhou et al. propose a pressure sensitive mat to detect location, but this type of sensor is not suitable

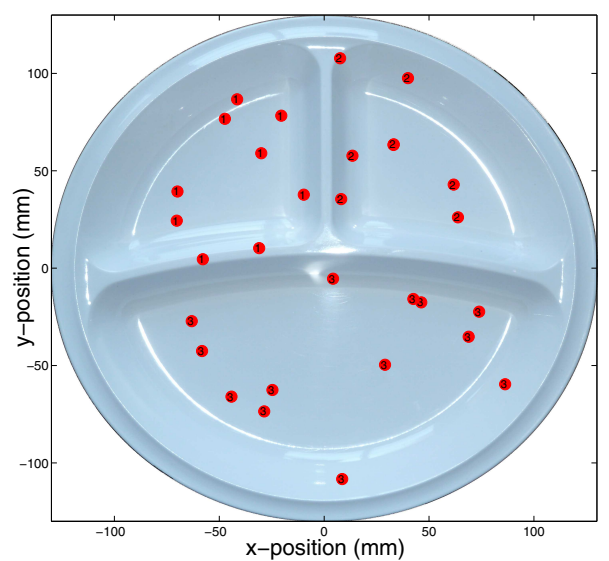

Fig. 5: The location of detected bites in the lab measurement.

to measure weight [8]. To increase the robustness of the smart plate in unrestricted environments, more data will be recorded in realistic environments. Furthermore, future work will focus on the process of step detection. This can be done by exploring different signal processing techniques and by combining data from different sensors such as wearable devices with the smart plate.

\section{REFERENCES}

[1] L. Burke, M. Warziski, T. Starrett, J. Choo, E. Music, S. Sereika, S. Stark, and M. Sevick, "Self-monitoring dietary intake: Current and future practices," Journal of Renal Nutrition, pp. 281-290, 2005.

[2] O. Amft, "A wearable earpad sensor for chewing monitoring," in Sensors, 2010 IEEE. IEEE, 2010, pp. 222-227.

[3] S. Passler and W.-J. Fischer, "Food intake monitoring: Automated chew event detection in chewing sounds," IEEE journal of biomedical and health informatics, vol. 18, no. 1, pp. 278-289, 2014.

[4] G. Mertes, H. Hallez, B. Vanrumste, and T. Croonenborghs, "Detection of chewing motion in the elderly using a glasses mounted accelerometer in a real-life environment," in Engineering in Medicine and Biology Society (EMBC), 2017 39th Annual International Conference of the IEEE. IEEE, 2017, pp. 4521-4524.

[5] J. Fontana, M. Farooq, and E. Sazonov, "Automatic ingestion monitor: A novel wearable device for monitoring of ingestive behavior," IEEE Transactions on Biomedical Engineering, pp. 1772-1779, 2014.

[6] R. Zhang and O. Amft, "Monitoring chewing and eating in freeliving using smart eyeglasses," IEEE journal of biomedical and health informatics, vol. 22, no. 1, pp. 23-32, 2018.

[7] H. R. Kissileff, G. Klingsberg, and T. B. Van Itallie, "Universal eating monitor for continuous recording of solid or liquid consumption in man," American Journal of Physiology-Regulatory, Integrative and Comparative Physiology, vol. 238, no. 1, pp. R14-R22, 1980.

[8] B. Zhou, J. Cheng, M. Sundholm, A. Reiss, W. Huang, O. Amft, and P. Lukowicz, "Smart table surface: A novel approach to pervasive dining monitoring," in Pervasive Computing and Communications (PerCom), 2015 IEEE International Conference on. IEEE, 2015, pp. 155-162.

[9] R. S. Mattfeld, E. R. Muth, and A. Hoover, "Measuring the consumption of individual solid and liquid bites using a table embedded scale during unrestricted eating," IEEE journal of biomedical and health informatics, vol. 21, no. 6, pp. 1711-1718, 2017.

[10] M. Puri, Z. Zhiwei, Y. Qian, A. Divakaran, and H. Sawhney, "Recognition and volume estimation of food intake using a mobile device," in Workshop on Applications of Computer Vision, 2009.

[11] H. He, F. Kong, and J. Tan, "Dietcam: Multiview food recognition using a multikernel svm," IEEE journal of biomedical and health informatics, vol. 20, no. 3, pp. 848-855, 2016.

[12] C. K. Martin, S. Kaya, and B. K. Gunturk, "Quantification of food intake using food image analysis," in Engineering in Medicine and Biology Society, 2009. EMBC 2009. Annual International Conference of the IEEE. IEEE, 2009, pp. 6869-6872. 